\title{
Poetic Images and Their Connotation as a Gateway to Inspire Design and Printing Artwork
}

\author{
Farida Shaban Mohamed. PhD \\ Associate Professor \\ Musaed Mohammad Beheiri.PhD \\ Assistant Professor \\ College of Education Public Authority for Applied Education and Training. \\ The State of Kuwait
}

\begin{abstract}
Although poems not only are the formation of technical words, Since the ancient times, but also, it has a common ground with the visual arts. Poems have a language that consists of vocabulary, construction elements, and poetic images. However, painting also has elements of the artwork and vocabulary, images, techniques, technical workspace ...etc.

On the other hand, different ways of expression are extended through two dimensions as such reality, imagination as well as the ability to translate sensations and feelings to aesthetic artistic curriculum templates.

For the sake of a design curriculum, the research problem tried to exploit the poems material as input to inspire the expressive imagination. The importance of the present research is as a poems translation and interpretation to be transferred to images and paintings within the aesthetic framework. This transferred imagination blends artistic culture of plastic arts with poems. The urgent need for openness between these fields will enrich each field and support creativity with new innovative variables. The research aims to analyses the relationship between printed artistic design and the art of poems through the translation of poem verses into spatial meanings of expressional features. However, it will create new artistic entries based on the breadth of the expressive range in the poetic process.

For creating new and innovative visions areas beyond reality and to trigger fiction and symbol, the present research assumed that it is possible to take advantage of the commonalities between poetic and art creativity. By using the water colored (Gouache) and the white paper surface (Canson), the search was limited by several expressional styles ranging from realism, abstract...etc., as well as applications on the fourth level of Applied Education and Training College of Education-The State of Kuwait.
\end{abstract}

Keywords: poems, visual arts, artistic curriculum, plastic arts.

\section{Introduction}

Generally, poems is the formation of a technical words could embody things, life and other assets, where it set out the mind and spirit to heights beyond the fact images. this embodiment is varied and extended across several levels where sometimes:

- Direct clarity,

- Hidden meanings or included,

- Realistic and abstract,

- Expressive and symbolic.

Here it could be recognised the harmony between poems and fine arts fields in general. It's an open opportunity to express all human feelings and emotions, which could reveal deep hidden feelings, awakened and crystallised out in words and poetic images as well as create elements, shapes and colors. There is also another advantage which more complicated than just create a sense of cognition. In different words a huge production and creation of upscale expressional images, renewable and infinity [1].

For the sake of the poems, we own:

- The language with its vocabularies,

- Elements of construction,

- Poetic images and techniques

- Rhetorical techniques,

- Elements of the artwork,

- Shapes ,Space and technology,

It's a creative art with a common ground and completely through the combination of artistic expression. [2].

By sketching or drawing, the idea of make a link between poems and drawing has been existed since the ancient times through the provision of meaning. In spite of the convergence and mutual influence between the two arts, each of them carries its distinguished specificity expressed by its own different tools [3]. 
Plastic arts are spatial in the first place, occupying space or area on emptiness. The place holds temporal features cross-references, connotations, elements at different ways of formulating. However they are largely limited keeps the place simulated. However, in the poetic work; every word can be a world unto itself. An image in a mind could be formed through visually linked to the cultural, social and historical dimensions. It is within the construction of poems, where construction includes the image, and the image within the overall context of the text. Here temporal is progressing the spatial. Maybe the spatial is formed according to the temporal of the poetic text and not as in the artwork where previously spatial progress on the temporal. However, the plastic artwork provides the recipient constitute a simi-complet product which takes a specific place by appointed within the perspective of the visual sight [4]. For the receiver imagination to the meaning rebuild, diversity with devoting a whole time and temporal breadth, will suggest a movement which so much required. The perceived, which are important values and an integral part of the reconstructive process in any plastic artwork in any of the fields of art design, will be interpreted by word or color or shape [5].

Researchers have noticed at the period of design and decoration course teaching (level 4), that students need a great effort to raise their fictional abilities from the fundamental points of convergence between the concepts of poetry and plastic artwork. From the above discussion, therefore, the idea of the present research generated.

whilst starts doing some creative designs and in spite of the art of poetry inspired in the artwork design and printing, but it could provoke imagination and stimulation which aren't limited and not final .On the other hand the idea of the present research is loaded with a variety of expressional energy through an extensive area of realism, symbolism, and even imagination.

However a question has raised up as could the poetic materials whatever, a poem or prose considered as introduction to inspire and stimulate the imagination?

Furthermore the poetic materials what so ever considered as aesthetic and expression within the decorated design course of the [5] faculty.

\section{V.2.The poetic image components: -}

V.2.1.Language component:

Where the language is the pillar of poetic image, the fabric of vocabulary

is a reflected image to expresses the poetic expression experience.

V.2.3.Imagination components:

The Imagination is the spirit vocabulary that takes a psychological

Cognitive template in the poet case.

\section{V.2.4.Imagination components:}

It's the language and the passion of image could identify to react with the receiver in form and content [2].on these types and components of the language of the time, which makes poems as well as the receiver aviate in the imagination of time to translate the feelings and emotions of the words. Whilst the expression in the language of plastic artwork associated with place, any work of art components of the product and its elements and the idea to be expressed in a manner or more of artistic styles, or modern and contemporary schools that emerged on the art scene.

A reflexive process in translating the feelings and emotions and result in a mental image of the artist in a human dynamic mind influenced by technical innovation. It will be the focus of this vulnerability on the significance and types, geared towards what is happening in the reader mind. In other words, as a result of the generated response by the image in the recipient mind, is sensually limited. It could be classified according to its visual images Article to audio and gustatory and olfactory. That is the image as a pattern embodies the vision of a symbolic.it combines real and metaphorical, or both of them as symbols derive their effectiveness from the collapsed psychological.it could reveal a significance symbolic vision by the poem or artwork. This type is the most basic of these connotations that are most likely to mind. [6].

\section{Hypotheses}

1. The Arabic words language poetry carries its own significance and structure, which withstand a wider connotations where could exceed the limits of reality.

2. There are many similarities between the concept of poetic and plastic art creativity.

3. It could utilise the commonalities between poetic creativity and the plastic art to create a new fields and innovative visions beyond reality towards the prospects and fictional symbolic implications. 


\section{Research aims}

1- To Monitor and analysis the relationship between printing art design and poetic art from being expressive, artistic and aesthetic.

2- Emphasis on the concept of symbolic expression and stimulate the imagination through the translation of words into the meanings of spatial with concrete impersonal features.

3- To Find a new artistic entries based on the breadth of the expressive range in the poetic artwork as realistic, imaginary, and symbolic.

4- To create an innovative artistic designs be adequate for printing.

\section{Research importance}

1. The research emphasis on the importance of creative thinking in the artwork associated with the word with its meaning and significance to be translated in values, aesthetics of artistic design, and printing.

2. The research concerned with the process of translation, interpretation and transform poems into images and temporal frame, spatial aesthetic within the framework of the two cultures of plastic-art and poetry-art.

3. The research deduct multiple technical inputs poses so as to achieve a combination of hearing, vision, imagination and sense,

4. This product forms an emotional trend where be tested by professionals and who are interested in art and poetry alike.

5. Research confirmed the urgent need for openness between the fields and each other to enrich each area and support with new innovative variables.

\section{Search limits}

1- The Research is an open art methods ranging from realism, abstract, expressionism, symbolic, Impressionism ... etc.

2- Reliance on ancient and modern poems whatever its prose poem or poetic images.

3- The research is applied on the Department of Technical Education girl's students who are studying the printing design course.

4- The implementation process depends on the gouache colors, ink printing, the white reinforced paper surfaces (Canson type), soft templates and stencil.

\section{Terminology}

IV.1.The concept of poetic creativity: -

- Poetic creativity is an openness vision and search for the aesthetic diversity of vision by construct an aesthetically wide range.

- Supported by bilateral dialectic relationship as absence /attendance, internal / external self-reliance, the body and the subconscious mind.

- It is determined by the races perception for poem concept in the light of the technical framework that reported by the poet who seeks to express his vision in accordance with the construction of textual free poem [2].

\section{IV.2.Abstract expressionism:-}

- Based on the theory that if the colors, fonts, shapes, have been used freely in the installation of an informal construction, or when used according to the official concepts or when used to represent thing, will be able to express delightful sight.

\section{IV.3.Symbolic expressionism:}

- From special element it would take an expression to make it conventions to denote on meaning known as articulation, phrases, announcement, and signal.

- The components of any artwork such as the texture or block, or a space.

- This expression becomes symbols of visible forms differ in their expression and accuracy from one community to another [7].

\section{IV.4.Realistic Expressionism:}

- It focuses on the objective direction, with the object as more hatred than itself.

- It's the daily life documentary with honesty and integrity.

- Written paper not have any feelings or expression of the sense of an artist, it is a tangible and visible to the reality of any activity of individuals. [8] 


\section{Research Methodology}

The research is dealing with the descriptive and analytical approach with theoretical Research in one of its aspects. Moreover, in the design and implementation of the search experience an experimental approach has been carried out as follows:

V.1.The Theoretical Approach and its parameters:-

1. Artistic expression philosophy.

2. What are the symbols and its role in highlighting the expressive meanings and connotations in the artwork?

3. The analysis poems usage with the artwork for some contemporary artists.

\section{V.1.1.Its therefor has to discuss these parameters}

\section{Artistic expression philosophy}

Expression is the result of the interaction of an idea whether it is objective, spiritual, mystical or cosmic, within a certain subject, with medium and materials, with the technical drafting, with the skills and techniques, or in the artist mind.

Expression contains numerous indications between their symbols and aesthetic significance; where through the artwork it could be disclosed. It is the lively nexus between the artist and his materials work surface in the process of artistic creation. It is a dimensions simulation of intangible and emotional process of the creative process explanation through the creative experience livelihood [1].

The artistic expression in the artwork of a single set associated with groups' relationships is divided into small groups of elements through the artwork. These divisions play a role as a reflection of an equivalent of the content to be conveyed through expression.in spite of the expressive connotation; it is broader than the expressions of the elements if they were separated. Although it could be said that the expression stems from being a subjective process expressively embodied in a physical or behavioral medium, but it could be explained on the surface of the artwork by the artistic techniques, methods, ways of drafting and creative vision [8].

\section{V.1.2. What is the symbols and its role in highlighting the expressive meanings and connotations in the} artwork?

Symbol is a means of expression. The technical Symbol has an aesthetic mean where it helps its interpretation and understanding, both in terms of colour meaning or chromatography significance. In the field of plastic art the icons usage could get different meaning in other areas.if we set the shape of symbols in different field, our understanding and our sense will be affected. However the changed Symbol form and its connotations depending on the applied area where a new expressional messages will be reached.it could has a gloss aesthetic in terms of its connotation as a color, shape and design or even form. In the artist's language, these symbols are to communicate with others and hence influence it to deliver a massage [8].

\section{V.1.3. The analysis poems usage with the artwork for some contemporary artists.}

A lot of artists have resorted to use the language and the word as a means of expressing artistic idea. To explain the implications of the sensory and emotions embodied in the encaustic, the usage whether in the form of words image or poetic images has to has a symbolic connotation. Artists have used different surfaces textures and materials to highlight the aesthetics of the written word, or poetry, or even a combination between them in an innovative artwork.

\section{VI.1.1.Sohrab Sepehri}

\section{Previous work}

The Iranian poet and a painter. The Portrait (1) is showing a painting portrait using acrylic and water color in a frame $(55 \times 35 \mathrm{~cm})$. Sohrab is expressing the importance of water which is the lifeblood and the trove secret of everything on this planet. The Poems therefore became part of the painting, where the artist magnified the word of water in a bold black color whilst the other words in a small letters emphasising on the AB (i the Persian word of water).

\section{VI.1.2.The portrait interpretation}

The Poet used the water in his painting portrait in the form of rivers flowing into each other. Through the portrait, the paint occupied a lot of spaces which drew on the form of flexible arches on two horizontal levels. The water is flowing from each of arches and go downstream to the other, by which irrigate the ground. The water word was colored in black indicating its importance and function in the life. However, together with the main lines he finished the rest of the poetic verse by curved line with a smooth movement. Eventually disappear within the background. 
With the blue color gradient, the artist chose the realist doctrine of the background as designed as combination between nature and mountains mist. This combination of water with the image, however, highlights of transparency of water conception in the painting portrait. Eventually the bud plant emerged between the water estuaries. The beautifulness of word was shining with the technical elements. However in connection between word and image in the artwork, the drawing and background interacted as well as the water function.

\section{VI.2.Ali Al-Padah}

The Kuwaiti painter

The portrait is one of watercolor techniques as rays and shadow moving out of the words at different paths to symbolize the earth, which led to the shape interact with the portrait ground .The name of portrait (2)is "God the Great' ' $(100 \times 100 \mathrm{~cm})$ with 18 karat gold plate. It is recognized from Portrait (2) that the word of God the great as started with the poetic verse and occupied a wider area in this portrait. Using the 18 karat gold metal, the artist's draw the word of the Majesty code to indicate and embodiment as His Majesty the Creator is above all.Hence to highlight the expressive value and the color of his majesty code he used the brown color on the background and then repeated the shadow of the code with a green color emphasizing on the shadow as the radiation of the golden color.

When the shadow takes the path under the Majesty code, it turns green sign as ground fertility. However, By using a set of colors as brown, yellow and beige in different degrees with texturized effects at the background of the work, the shape and type of line indicates and demonstrates that the keyword the Creator, resort in everything.

The Islamic decorations at the bottom right of the portrait denote that the Arab Islamic decoration is connected with ancient history. It be could recognized that his Majesty code, 'the Creator' with its shadow brought a positive and attractive movement.

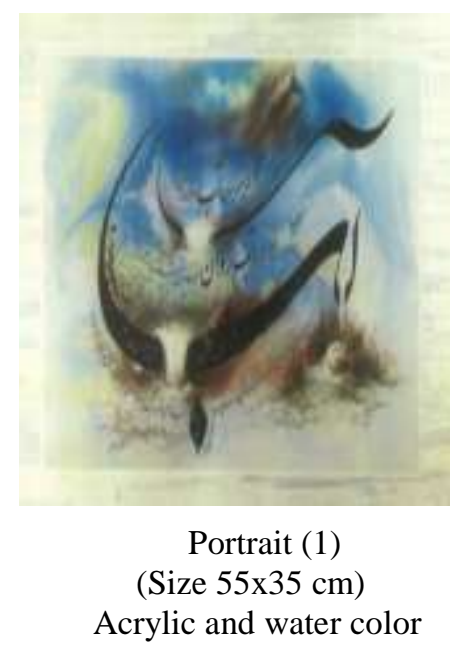

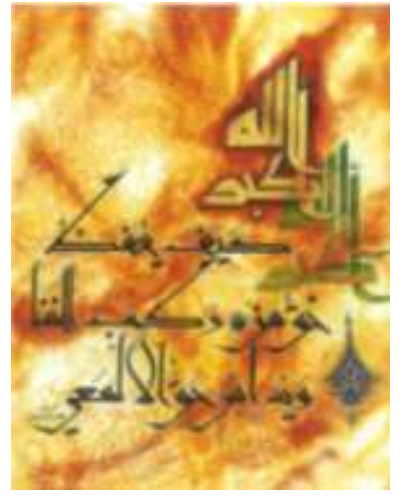

Portrait (2)

(Size 100x100 cm)

18 karat gold plate

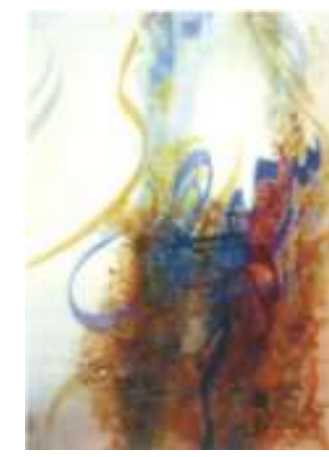

Portrait (3)

(Size 100x120cm)

acrylic color

\section{VI.3. Khalid El Saei}

The Syrian painter has drawn this portrait as nominated as Unlimited Professionallity. The artist used the abstract style of art in his portrait through the quotation elements of the Arab civilization by using the Arab intellectual letter where he exploit the texts of the Son of Muqlid, the Son of Pawab, Rumi and other poets in contemporary history. In the portrait (3) it's recognised that that the painter set himself free from the strict line rules towards the vision of his own Arab calligraphy. The technique is based on the color formations aesthetics of with small and large overlapping using the technique of color, line, shape. However, He broke the traditional geometry of the line. Moreover, established a new relationship between the individual words and its meaning whilst merged the painted line with color, mass and spaces .It could be seen spontaneous letters coup across a fast rhythm of a musical tones. The portrait depends on a musical lines reference such as jazz and blues rhythmic. On the other hand the gray color with a little reddish color accomplish with monolithic lettering formulate on a light background an alphabet. The concept of the portrait transferred from classical calligraphy toward cosmic modernity celebrates with abstract style. However the concerns core of the artist in his architecture portrait and layers of color blends the dust with the contemporary life rhythms gave indication of contradiction and contrast. The portrait stored an emotional charge and deductions color with decorative elements to the rhythm of the poem itself. 


\section{VI.4.The foundations of the design structural:}

The design structural foundations take an important role in the idea planning process and built the structural system of the design, which consists of the elements and form of language vocabulary used by the artist designer. On the other hand so named with refer to flexible possibilities to choose any flexible form and its usability of integration and harmony with each other to build an entirely technical work form.

The nature of the design whether color linear, formal and silhouette does not depend on its shapes, form as well as its impact in the void space. Moreover it connect with the visual appearance which regulated by these elements and building a flat plastic art modalities. However this is through the total performing operations organized by the design process.

The role of each element in the construction of plastic art work has to be confirmed by the structural foundations. Not only that but also its impact which influenced by the elements surrounding it.This roles include the plastic elements of one of its patterns of interconnection systems between each other.

All of these principles and elements play an important role in highlighting the concept of expression, interaction and a sense of the elements and to show what is going on around the idea of the artwork by the human internal Webmasters. [5]

\section{The Plastic Artwork Aesthetic Images As A Technical Art Relationship With The Poetic Images}

It's known that the image has the technical elements such as fact, thought, emotion, and imagination where the fields of artwork and poetry are shared. All these elements nourish the artistic image of the artist, whether in poetry or artwork .these technical art embodied in the sensory stuff that could be perceived in the process of expression. This means that the expression is a term that translates sensations, emotions and imagination in an artistic models in spite of that the expression influenced by the social reality and the modalities of figurative and expressive artistic dimensions but the artist seeks to achieve and disseminate.

Technical image is considered a form of global reflection. Then the revelation of the idea of the artwork comes including the intellectual storage carried by the artist within the artwork. However when the idea stands out in the image on the other elements, it raises the value of the work aesthetically and move into another cognitive relationship. The last component of emotion must synchronies with the experiment, and interferes with vision to take its vital role in creating the image [10].

The psychologists were linked the unconscious process creativity, with the component of vital subconscious in the technical image. Although the stored subconscious at various forms provoked by contemporary and realistic events, but the Imagination on the other hand is the common component key to all the component of arts. So the Imagination evokes the raw material for the image and selecting elements that would be form the image later, and emerging them together even to lose all the its features. These elements therefore are shown in the form entirely different from what it was. However the methods of expression in poetry are based on the word that shaped by the poem images [10].

\section{V.1.Types and components of the poetic image:}

V.1.1.Single Image

That rely on imaging sensory exists between similarly apparently without access to psychological meanings. V.1.2.Composite Image

It formed by imaging combines what is sensuous and what is psychological passionate with overlapping elements.

\section{V.1.3.The Composite images}

It depends on the concentration of all the elements.

V.1.4.The overall image:

It depends on the concentration of all elements of the image through the coordination in the context at one expressive, combining what is holographic and what is psychology, in the form reflects the experience of the poet [6].

\section{Experiment work}

In order to translate words into aesthetic meanings with the same expressional features and by the overall objective of creative experience in the application of innovative designs, it was recommended to make sure the relationship between poetry and art through symbolic connotations and imagination style are related. To achieve this target, it was carried out by the Girls students design and decoration of the 4th level department of the College of Basic Education. The necessary sketches had been prepared to illustrate the idea of the Experiment. 
VI.1. the limits of experience:

- Testing for preliminary designs for the idea by using a pencil and then colored with wooden color as a first stage, and then magnifies the design over a large area.

- Use gouache printing inks, paper and Canson paperboard measuring $(100 \times 80 \mathrm{~cm})$, printing paper, soft templates, stencil

- The poem will be used as a separate work whilst by the artwork components and its structural foundations, the poetic verse will be translated as a printing designed portrait, so as to allow an expressional wide space where the elements and shapes be expanded.

VII. An analytical study of an experimental model that illustrates the idea of contact the image in its stages with the poetic text

- display the poetic Verse

- A description of the artwork and its translation in accordance with expressive generated connotation by the structural design foundations of the words.

The following model as an example of experiment:

VII.1.Work characterization:

- The expressional artwork trend:

The artwork has styled the symbolic and visual imaginary.it highlight the idea by represented the eye, tears, and the faces inside the design elements with the ground and staircase in the center of the artwork of the portrait.

\section{VII.2. Degree of expression:}

- It's clearly shown by the eye and tears as well as the expressive faces of different expressions at the top and bottom of the painted portrait, where the meaning of words concluded inside the tears form. Portrait (4)

VII.3. The nature of the elements:

- Geometric and organic elements have been used marked by the staircase and by which is represented in all the design elements and distributed between figure and ground.

- Linear color Geometric areas at the work bottom which splits into two divisions, one right and own left.

- The linear areas interfere in a relationship in the form of an oval in various sizes and descend down to interact with the ground at the work bottom.

- Every oval shape contained a specific code to the words of poetic verse.

- A form of the transparent human element emerged in the center of the design while the forms are distributed as faces of different people in a variety of expressions in the design ground .

\section{VII.4. Form and ground relationship}

- Design elements were taken as interactive relationship and complementarily between figure and ground.

- The other half of the poetic verse took the meaning of death and hereafter shaped as faces in the present stages.

- At different levels the portrait Ground interfere with the original design in colored and formal relationships whilst interact with the design to complete the poetic verse.

- The portrait Ground became an active and equal part with the design for the miscellaneous symbols to express the poetic verse.

VII.5. The paths movement between the elements and its situation where the design depends on the following features:

- The tears Movement and its falling spreaded all over the painting portrait expressing the meaning of crying.

- The tears flowing movement headed down telling the softness expression and quietly slowing down directly on the portrait ground.

- The concept of linking life and death is shown as the frictional tears as if not breaking down, but merged with the dusty background.

- According to its situation on the design, the faces movement and its expressions between stillness and tranquility chose expressively metaphorical meaning.

- The faces met in different directions and sets at the bottom of the portrait reflecting the meaning of the death.

- The feature of the faces in the design center is expressing happiness in this world.

- The happy faces eventually as ascend to the top, changed its returning path with those expressions. 
- The staircase movement, which began from the design center and climb to the top is divided into two sections: section heading to the right, which represents the bulk of the design, carrying the eye and tears with the world, while the other section is moving to the left carrying the tears and its movement to the bottom represented death and the afterlife.

The color divided the Design into three levels:

\section{Color levels}

The first level is Portrait (4):

- The staircase was divided into two sections as a left and right part indicating for good and evil, expressed in green.

- The left part of the staircase went to the right direction which the blue, pink, and green color came out.

- The left division with brown colored as the symbol of dryness as departed from the goodness to the death stage.

The second level:

The light colors (gray, beige, blue) of the faces at the top of the painting portrait, Portrait (5), represented as symbol of pure hearts as well as good deeds of expression.

\section{The third level:}

- Faces Located at the bottom of the painting portrait as emerged from dark colors indicating the Earth and Death.

- The colors on the Background emerged in the center of the right and left design between pink and gray as indication of the top and bottom connection.

- These positions of colors by the structural sequence in the design represent the poetic meaning.

- In light colors start at the top of the design to interfere and become darkened on its way down until ended to the color of the dust.

1. The eye:

\section{The used Symbol- Portrait (4)}

- $\quad$ By the construction process, the eye position in the right of the design represents the human soul symbol.

- $\quad$ Eye therefore took a lot of space in the design in order to express what human could see in this life which is expressed by the blue, green and red color.

- The eye that sees everything and control it for decisions making in human life.

2. Tears: by leaving the pleasures of life, as well as the good and evil as a Symbol and sign of remorse and regret.

3. Faces: The symbol of the life process of growth and stability at the afterlife.

4. Staircase: A sign of growth and passing over life stations until access to the afterlife.

Portrait $(4,5,6,7,8)$ and slide $(1,2,3,4,5)$ :

\section{X- Poetic Text Relationship with The Image}

- The human soul was expressed in this work by the transparent humans standing in front of the staircase, but the viewing by this level is always expressed through the eye. Portrait (4).

- Expression of building good and evil lies in the fission of staircase from the top to the right and left of the design.

- Leaving the happiness of the life Expressed in the tears and its sizes of components at the right hand, which contained construction process: palaces, flowers, social life, and emotions?

- The Hereafter habitat metaphorically linked to the concept of philanthropy in the life with the drops of tears components in one side.

- The evil work linked with the components of tears drops on the other side, which is expressed in the dryness and death.

- The meaning purport of the Verse of poetry is to ensure the working good dwelling and Goodness, and not be guaranteed in the event of the existence of evil.

- All souls leave this life which were represented in the center of the design to go down and buried under the ground.

- There are good souls rise and ascend to the top while the bad soul remaining in the agony life.

- This is noticeable in the expressions of the faces at the bottom of the work in Mystery.

- Eventually therefore these portraits could leave the question as such: - Do you think there is any option to specify the crossroads between the life and the hereafter or leave it for a time. 


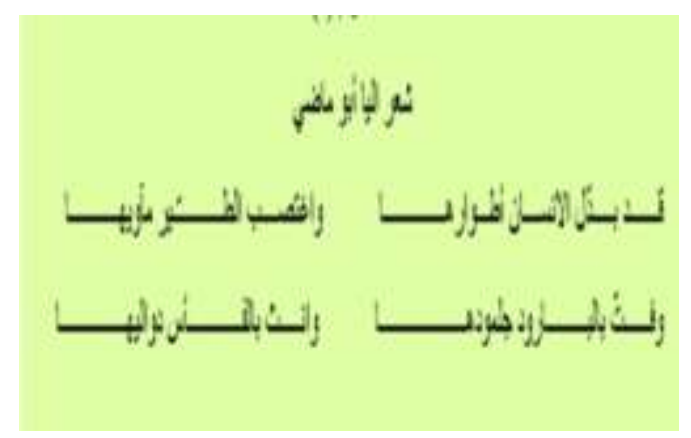

slide1

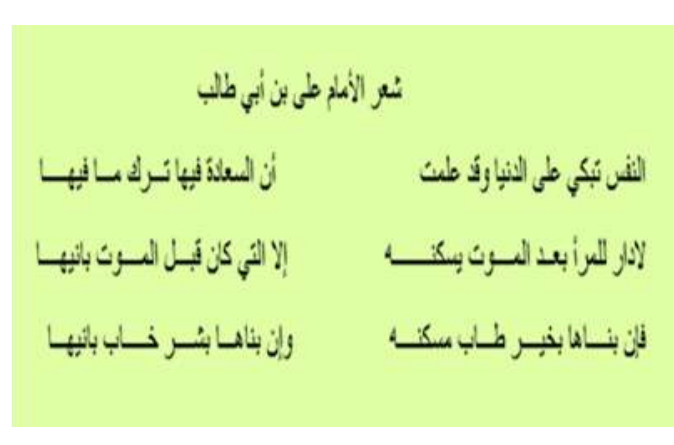

Slide2

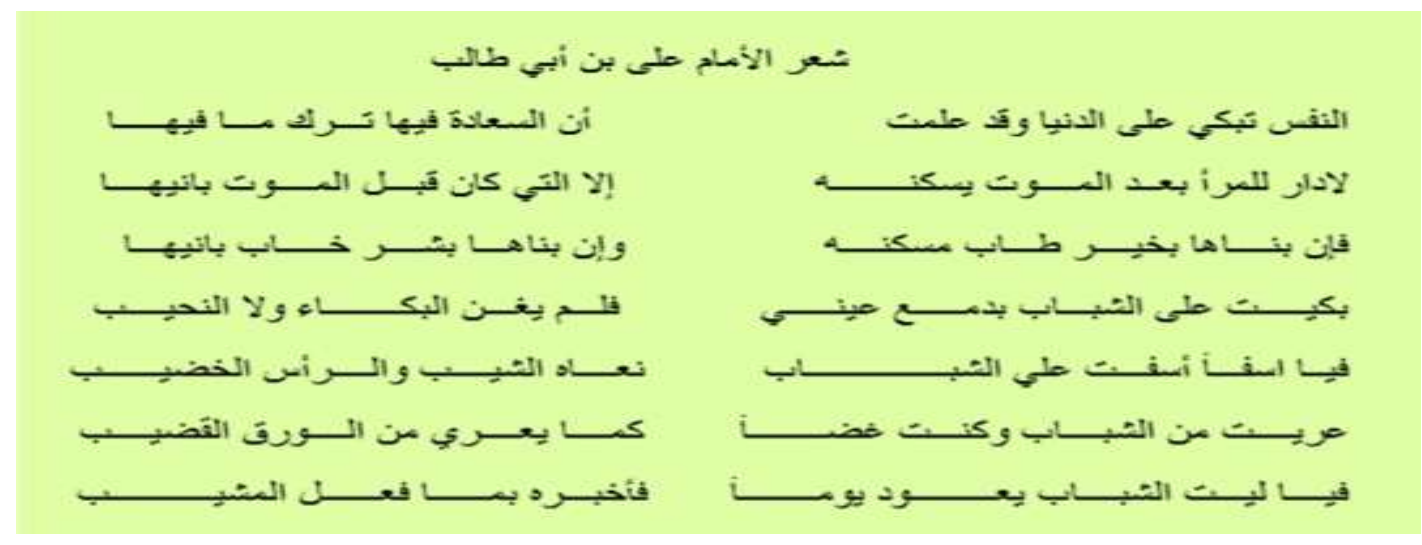

slide3

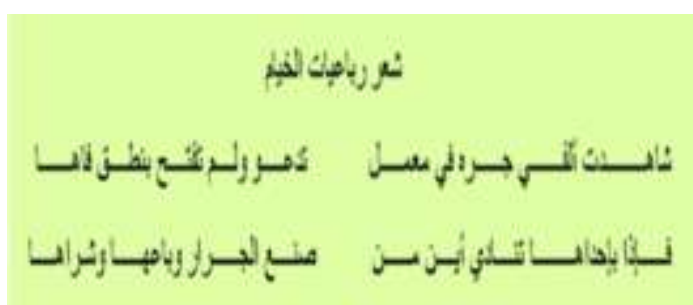

slide4

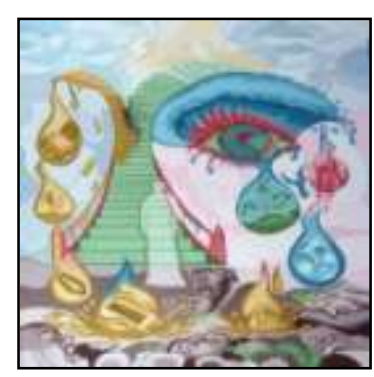

Portrait (4)/ slide2

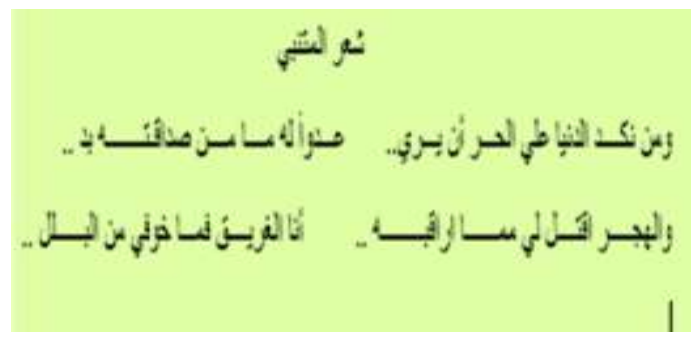

slide5

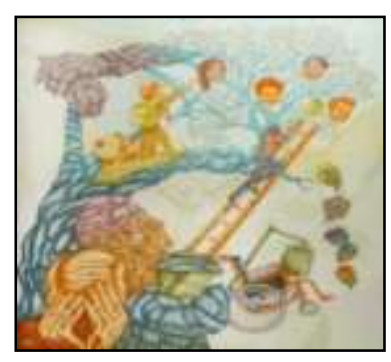

Portrait (5) /slide3

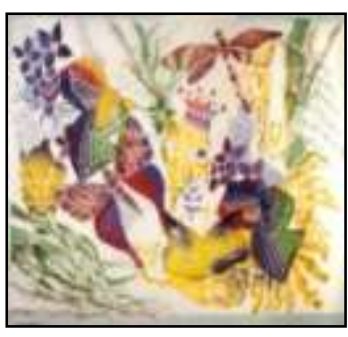

Portrait (6)/ slide1 


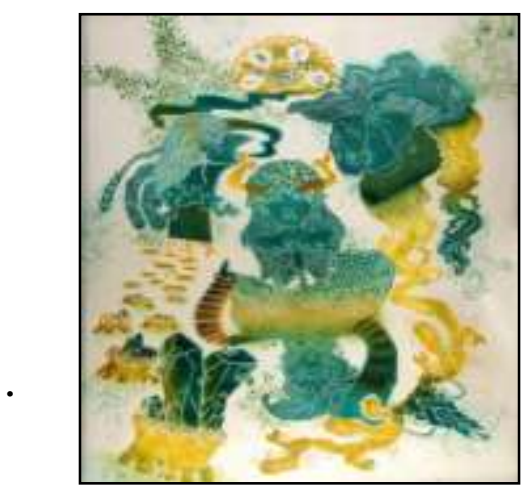

Portrait (7) /slide5

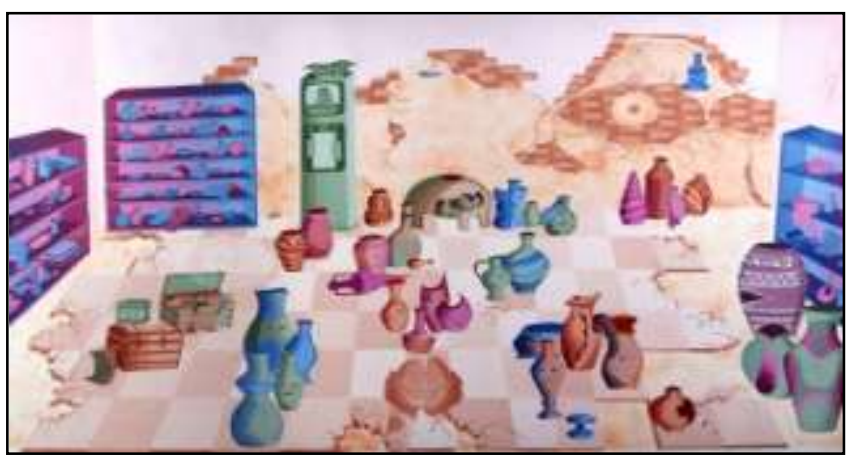

Portrait (8) /slide4

\section{Conclusion}

At the level of structural relationships in design, Expressive and symbolic aspect of the artwork is considered as the unfinished path of processes of innovation and open unlimited areas to savor other artists in different contexts.

- $\quad$ There is a close relationship between image and the expressive word meanings highlighted by the artwork, whether spatial or temporal arts.

- About symbolic and imaginary prospects, there are common denominators between the two areas which exceed the vision of reality.

- In poetic language with extensible ability for descriptive meaning in paintings innovative portrait, there are profound connotations.

\section{References}

[1]. The Jabra, J. (2000) art and artist. Beirut. Arabic Foundation for studies and publication

[2]. Eid.Y (1990) Techniques of narration in light structural approach .., 1, p 18. Beirut, Dar Al Farabi ISBN 978-9953-71-599-5

[3]. Azghai, A.Alshardodi, F .(2016) poetic between writing and Formation. Modern enterprise for culture and literature.

[4]. Obaid, C. (2010) aesthetic image in the dialectical relationship between plastic art and poetry Beirut. The magd.the University Corporation for Studies, Publishing and Distribution.

[5]. Zinedine, Th. (2012) literature and art: inspired plastic art in poetry. Damascus, the United Foundation for Press and Printing..

[6]. Heidegger, M(1927) Being and Time . New York: State University, Scm Press, New York Press .

[7]. Navadi, D. (2014) philosophy of abstraction in modern art. Arab Group for Training and Publishing

[8]. Mohammed, F.(2015) the diverse expressive connotations confirmation artwork through HTTP to enrich the graphic surface aesthetically and expressively in Holistic Thinking. Thinking processes. Specific Education Research Journal, Mansoura University, issu. (40)

[9]. Shawki, I. (2003) The Design: elements and basic in the plastic art. Cairo, Zahraa Middle Press.

[10]. Yassin, A. (2010), a poetic interpretation of the plastic art painting. Department of Culture, a magazine Tributary. www.arrafid.ae>arrafid.com 